01

\title{
О внутридопплеровской спектроскопии оптически возбужденных атомов в тонкой газовой ячейке
}

\author{
(C) А.Ч. Измайлов \\ Институт фризики НАН Азербайджана, \\ AZ-1143 Баку, Азербайджан \\ e-mail: azizm57@rambler.ru
}

Поступила в редакцию 23.01.2018 г.

Продолжено теоретическое исследование возможностей известного метода пробного монохроматического светового пучка при оптической накачке атомов (молекул) разреженной газовой среды широкополосным излучением в тонкой ячейке, диаметр которой гораздо больше ее внутренней толщины. В отличие от расчетов предыдущих публикаций по данному методу спектроскопии в настоящей работе рассмотрены произвольные значения интенсивности излучения накачки и толщины цилиндрической газовой ячейки. Таким образом, проанализированы всевозможные механизмы и особенности скоростной селекции атомов на оптически возбужденных уровнях, обусловленные пролетной релаксацией данных атомов в подобных тонких ячейках. На этой основе исследованы внутридопплеровские резонансы поглощения пробного светового пучка на квантовых переходах из верхнего уровня, возбужденного оптической накачкой. Полученные результаты могут найти применение в спектроскопии высокого разрешения атомов (молекул), а также для стабилизации лазерной частоты по установленным узким спектральным резонансам.

DOI: $10.21883 /$ OS.2018.06.46072.23-18

\section{Введение}

Для спектроскопии высокого разрешения очень важна разработка эффективных методов, которые позволяют анализировать структуру спектральных линий, скрытую допплеровским уширением вследствие движения атомов или молекул газовой среды [1]. Данные методы используются также для создания и оптимизации оптических стандартов частоты [2]. В работе [3] был предложен новый метод внутридопплеровской лазерной спектроскопии, основанный на стационарной оптической накачке основного атомного терма в течение пролета атомов достаточно разреженного газа между стенками тонкой газовой ячейки, у которой характерный поперечный размер $R$ гораздо больше, чем ее внутренняя толщина $L$. При этом световые пучки накачки и пробного излучения могут распространяться через такую ячейку даже в ортогональных направлениях, как показано на рис. 1. Впоследствии данный метод был успешно реализован экспериментально для прецизионного спектрального анализа и стабилизации лазерной частоты на основе серии тонких ячеек, содержащих пары́ цезия [4-6,8,9] или рубидия [7]. В отличие от широко используемой спектроскопии насыщенного поглощения в обычных „макроскопических“ ячейках (где $L>R)$ [1] в спектроскопии тонких газовых ячеек не возникают перекрестные резонансы на промежуточных частотах между центрами оптических переходов. Кроме того, соответствующие системы стабилизации частоты гораздо меньше подвержены влиянию частотных флуктуаций излучения накачки (которая может быть даже широкополосной), так как скоростная селекция оптически накачанных атомов определяется главным образом геометрией тонкой ячейки. Например, авторы экспериментальных работ [4-9] использовали цилиндрические ячейки из пирексного стекла (содержащие разреженные пары́ цезия или рубидия) с радиусом $R$ около $15 \mathrm{~mm}$ и различными внутренними толщинами $L$ от 0.5 до $10 \mathrm{~mm}$. В этом случае релаксация оптически накачанных (основного или метастабильного) атомных уровней обусловлена главным образом столкновениями атомов с торцевыми (плоскопараллельными) стенками ячейки. Поэтому оптическая накачка и пролетные эффекты в подобной тонкой газовой ячейке (рис. 1) приводят к неравновесному распределению оптически накачанных атомов по проекции их скорости $v_{z}$ вдоль ячейки (ось $z$ ) в области достаточно малых значений $\left|v_{z}\right|$. Таким образом, внутридопплеровские резонансы появляются в спектре поглощения пробной монохроматической волны с волновым вектором $\mathbf{k}$ (распространяющейся вдоль оси $z$ ) на центральных частотах оптических переходов из данных накачанных долгоживущих состояний в какие-либо возбужденные атомные уровни.

В рассматриваемом методе осуществляется оптическая накачка на электродипольном переходе $a \rightarrow b$ из подуровня $a$ основного терма атома на некоторый его возбужденный уровень $b$. Ранее при теоретических расчетах в работах [3-9] интенсивность такой накачки полагалась настолько низкой, что заселенность верхнего уровня $b$ была пренебрежимо мала по сравнению с населенностью долгоживущего состояния $a$. Поэтому в данных работах анализировались внутридопплеровские резонансы в поглощении пробного светового пучка на прямых квантовых переходах только из подуровней основного атомного терма. Вместе с тем в кратком 


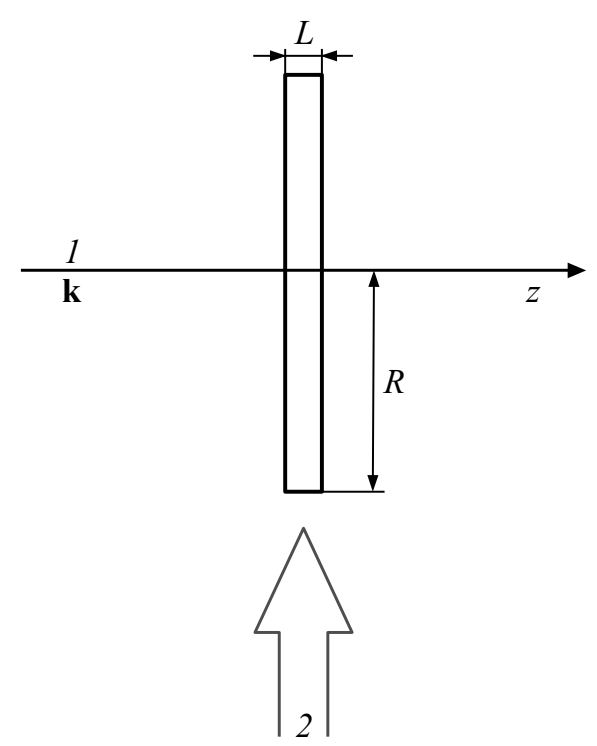

Pис. 1. Схема эксперимента с тонкой газовой ячейкой $\mathrm{c}$ радиусом $R$ и внутренней толщиной $L \ll R$, где $1-$ пробный монохроматический световой пучок, 2 - излучение широкополосной оптической накачки.

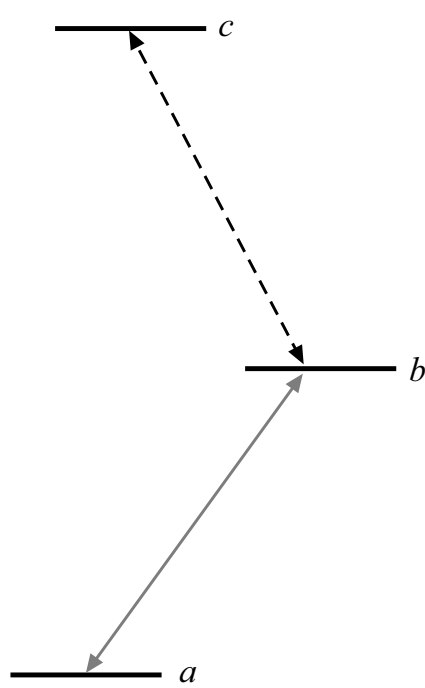

Рис. 2. Схема рассматриваемых квантовых уровней и переходов: $a \rightarrow b-$ переход из основного терма $a$, на котором осуществляется оптическая накачка атомов широкополосным излучением, $b \rightarrow c-$ переход, зондируемый пробным монохроматическим световым пучком.

сообщении [10] были представлены экспериментально зарегистрированные резонансы поглощения на квантовом переходе $6 P_{1 / 2}-6 D_{3 / 2}$ (с длиной волны $\left.876 \mathrm{~nm}\right)$ из оптически возбужденного уровня $6 P_{1 / 2}$ атомов цезия в ультратонкой газовой ячейке (с внутренней толщиной $L=10 \mu \mathrm{m}$ ), облучаемой широкополосным излучением накачки на переходе $6 S_{1 / 2}-6 P_{1 / 2}(894 \mathrm{~nm})$ из основного квантового терма цезия $6 S_{1 / 2}$. Это соответствует схеме на рис. 2, где детектируется поглощение на переходе $b \rightarrow c$ из уровня $b$, возбужденного при оптической накачке на переходе $a \rightarrow b$.

В связи с вышесказанным в настоящей работе исследованы внутридопплеровские резонансы поглощения на таких переходах $b \rightarrow c$, обусловленные особенностями скоростной селекции атомов на квантовых уровнях $a$ и $b$ (рис. 2) в тонкой газовой ячейке. При этом в отличие от расчетов предыдущих работ [3-9] не использовались ограничения на интенсивность широкополосной оптической накачки на переходе $a \rightarrow b$ (рис. 2) и толщину $L$ тонкой газовой ячейки (рис. 1).

\section{Основные соотношения}

Рассмотрим стационарную оптическую накачку атомов (или молекул) газовой среды широкополосным излучением в цилиндрической газовой ячейке (рис. 1). Предполагается, что в данной достаточно разреженной газовой среде можно пренебречь межатомным взаимодействием. Слабая пробная монохроматическая волна с волновым вектором $\mathbf{k}$ и сравнительно малым диаметром $d \ll R$ распространяется через центр ячейки вдоль оси $z$ (рис. 1). Частота $\omega$ данной волны сканируется в окрестности центра $\omega_{0}$ некоторого исследуемого квантового перехода $b \rightarrow c$ из оптически возбужденного состояния $b$ на верхний уровень $c$ (рис. 2). В то же время излучение широкополосной накачки действует на электродипольном переходе $a \rightarrow b$ из основного атомного терма $a$ в состояние $b$. При рассматриваемых условиях на основе известных балансных соотношений [1] получаем следующую систему уравнений для населенностей $\rho_{a}(\mathbf{r}, \mathbf{v})$ и $\rho_{b}(\mathbf{r}, \mathbf{v})$ атомов на уровнях $a$ и $b$ со скоростью $\mathbf{v}$ и координатным вектором $\mathbf{r}$ :

$$
\begin{gathered}
\mathbf{v} \frac{\partial \rho_{a}}{\partial \mathbf{r}}=\xi_{a b}\left(\rho_{b}-\rho_{a}\right)+A_{b a} \rho_{b}, \\
\mathbf{v} \frac{\partial \rho_{b}}{\partial \mathbf{r}}=\xi_{a b}\left(\rho_{a}-\rho_{b}\right)-\gamma_{b} \rho_{b},
\end{gathered}
$$

где $\xi_{a b}-$ вероятность светоиндуцированного перехода $a \rightarrow b$ (рис. 2), $\gamma_{b}$ - константа радиационного распада уровня $b, A_{b a}$ - вероятность радиационного распада по каналу $b \rightarrow a\left(A_{b a} \leq \gamma_{b}\right)$. Уравнения (1), (2) должны быть дополнены граничными условиями, которые зависят от особенностей атомных столкновений со стенками ячейки. Как и в предыдущих расчетах [3-9], выполненных для тонких газовых ячеек, будем полагать, что в результате таких столкновений устанавливается равновесное распределение как по скоростям атомов, так и по населенностям их квантовых уровней. Тогда из системы линейных дифференциальных уравнений (1), (2) получаем следующие выражения для населенностей $\rho_{a}(\mathbf{r}, \mathbf{v})$ и $\rho_{b}(\mathbf{r}, \mathbf{v})$ на уровнях $a$ и $b$ атомов в области пробного светового пучка, сконцентрированного около централь- 
ной оси ячейки $z$ (рис. 1) в интервале $-0.5 L \leq z \leq 0.5 L$ :

$$
\begin{aligned}
& \rho_{a}\left(z, v_{z}, v_{r}\right)=n_{a} F_{l}\left(v_{z}\right) F_{r}\left(v_{r}\right) \\
& \times\left\{\left[p\left(\frac{z+0.5 L}{v_{z}}\right) \eta\left(\frac{R v_{z}}{v_{r}}-z-0.5 L\right)\right.\right. \\
&+\left.p\left(\frac{R}{v_{r}}\right) \eta\left(z+0.5 L-\frac{R v_{z}}{v_{r}}\right)\right] \eta\left(v_{z}\right) \\
&+ {\left[p\left(\frac{z-0.5 L}{v_{z}}\right) \eta\left(z-0.5 L-\frac{R v_{z}}{v_{r}}\right)\right.} \\
&+\left.\left.p\left(\frac{R}{v_{r}}\right) \eta\left(\frac{R v_{z}}{v_{r}}-z+0.5 L\right)\right] \eta\left(-v_{z}\right)\right\}, \\
& \rho_{b}\left(z, v_{z}, v_{r}\right)=n_{a} F_{l}\left(v_{z}\right) F_{r}\left(v_{r}\right) \\
& \times\left\{\left[s\left(\frac{z+0.5 L}{v_{z}}\right) \eta\left(\frac{R v_{z}}{v_{r}}-z-0.5 L\right)\right.\right. \\
&\left.+s\left(\frac{R}{v_{r}}\right) \eta\left(z+0.5 L-\frac{R v_{z}}{v_{r}}\right)\right] \eta\left(v_{z}\right) \\
&+\left[s\left(\frac{z-0.5 L}{v_{z}}\right) \eta\left(z-0.5 L-\frac{R v_{z}}{v_{r}}\right)\right. \\
&\left.\left.+s\left(\frac{R}{v_{r}}\right) \eta\left(\frac{R v_{z}}{v_{r}}-z+0.5 L\right)\right] \eta\left(-v_{z}\right)\right\},
\end{aligned}
$$

где

$$
\begin{gathered}
p(t)=\left(\lambda_{2}-\lambda_{1}\right)^{-1}\left[\left(\xi_{a b}+\lambda_{2}\right) \exp \left(\lambda_{1} t\right)\right. \\
\left.-\left(\xi_{a b}+\lambda_{1}\right) \exp \left(\lambda_{2} t\right)\right], \\
s(t)=\frac{\left(\xi_{a b}+\lambda_{1}\right)\left(\xi_{a b}+\lambda_{2}\right)}{\left(\xi_{a b}+A_{b a}\right)\left(\lambda_{1}-\lambda_{2}\right)}\left[\exp \left(\lambda_{2} t\right)-\exp \left(\lambda_{1} t\right)\right], \\
\lambda_{1,2}=-\xi_{a b}-0.5 \gamma_{b} \pm\left[0.25 \gamma_{b}^{2}+\xi_{a b}\left(\xi_{a b}+A_{b a}\right)\right]^{0.5},
\end{gathered}
$$

$\eta(x)$ - ступенчатая функция $(\eta(x)=1$, если $x \geq 0$, и $\eta(x)=0$, если $x<0), n_{a}$ - равновесная плотность атомов на уровне $a$ c распределениями Максвелла $F_{l}\left(v_{z}\right)$ и $F_{r}\left(v_{r}\right)$ соответственно по продольной $v_{z}$ и радиальной $v_{r}$ компонентам атомной скорости $\mathbf{v}$ :

$$
\begin{gathered}
F_{l}\left(v_{z}\right)=\pi^{-0.5} u^{-1} \exp \left(-v_{z}^{2} u^{-2}\right), \\
F_{r}\left(v_{r}\right)=2 v_{r} u^{-2} \exp \left(-v_{r}^{2} u^{-2}\right),
\end{gathered}
$$

где $u$ - наиболее вероятная скорость атомов в газе.

Из выражений (3) и (4) получаем следующие зависимости от продольной компоненты скорости $v_{z}$ усредненных населенностей $N_{a}\left(v_{z}\right)$ и $N_{b}\left(v_{z}\right)$ уровней $a$ и $b$ оптически накачанных атомов, сосредоточенных около центральной оси ячейки $z$ (рис. 1):

$$
\begin{gathered}
N_{a}\left(v_{z}\right)=L^{-1} \int_{-0.5 L}^{0.5 L}\left[\int_{0}^{\infty} \rho_{a}\left(z, v_{z}, v_{r}\right) d v_{r}\right] d z, \\
N_{b}\left(v_{z}\right)=L^{-1} \int_{-0.5 L}^{0.5 L}\left[\int_{0}^{\infty} \rho_{b}\left(z, v_{z}, v_{r}\right) d v_{r}\right] d z .
\end{gathered}
$$

Заметим, что результаты исходной теоретической работы [3] вытекают из соотношений (3)-(10) при следующих ограничениях на внутреннюю толщину газовой ячейки $L$ и интенсивность широкополосной оптической накачки:

$$
L \gg u / \gamma_{b}, \quad \xi_{a b} \ll \gamma_{b} .
$$

При ограничениях (11) населенности уровней (9), (10) связаны соотношением $N_{b}\left(v_{z}\right) \approx\left(\xi_{a b} / \gamma_{b}\right) N_{a}\left(v_{z}\right)$.

Коэффициент поглощения $G$ слабой пробной волны на переходе $b \rightarrow c$ (рис. 2) в рассматриваемой газовой ячейке имеет вид

$$
G(\delta)=G_{0} \frac{\left\{\int_{-\infty}^{\infty} N_{b}\left(v_{z}\right)\left[\gamma^{2}+\left(\delta-k v_{z}\right)^{2}\right]^{-1} d v_{z}\right\}}{\left\{\int_{-\infty}^{\infty} 0.5 n_{a} F_{l}\left(v_{z}\right)\left[\gamma^{2}+\left(k v_{z}\right)^{2}\right]^{-1} d v_{z}\right\}},
$$

где населенность $N_{b}\left(v_{z}\right)$ определяется формулой (10), $\delta=\left(\omega-\omega_{0}\right)-$ отстройка частоты пробной волны $\omega$ от центра $\omega_{0}$ исследуемого перехода $b \rightarrow c$ с однородной полушириной $\gamma$ спектральной линии, $G_{0}-$ значение коэффициента поглощения на данном переходе $b \rightarrow c$ при нулевой расстройке $\delta=0$, полученное в предположении, что заселенность оптически возбужденного уровня $b$ достигнет половины от равновесной плотности атомов $n_{a}$ в исходном основном состоянии $a$ (рис. 2).

\section{Обсуждение результатов}

В отличие от ограничений (11) в расчетах предыдущих работ [3-9] мы проведем анализ на основе полученных соотношений (3)-(10) для произвольного параметра насыщения $\left(\xi_{a b} / \gamma_{b}\right)$ оптического перехода $a \rightarrow b$ (рис. 2), а также при следующем условии:

$$
L<u / \gamma_{b}
$$

Такая ситуация может иметь место для достаточно долгоживущего оптически возбужденного уровня $b$ (рис. 2) или при очень малой внутренней толщине $L$ газовой ячейке (рис. 1). В частности, условие (13) выполнено в отмеченном выше эксперименте [10], где реализован рассматриваемый метод спектроскопии на переходе типа $b \rightarrow c$ в ультратонкой ячейке $(L=10 \mu \mathrm{m})$ с разреженным паром цезия.

На рис. 3 представлены зависимости эффективных населенностей $N_{a}\left(v_{z}\right)$ и $N_{b}\left(v_{z}\right)(9),(10)$ оптически накачанных атомов на уровнях $a$ и $b$ (рис. 2) от их продольной компоненты скорости $v_{z}$ при достаточно высоких интенсивностях широкополосной оптической накачки и различных отношениях $R / L \gg 1$ радиуса $R$ цилиндрической газовой ячейки к ее внутренней толщине $L$ (рис. 1$)$. При этом рассмотрены случаи как нециклического, так и замкнутого перехода $a \rightarrow b$, когда в уравнениях (1), (2) $A_{b a}=\gamma_{b}$, т. е. уровень $b$ может радиационно распадаться только в основное состояние $a$. Одинаково нумерованные зависимости на рис. $3, a$ и $3, b$ отличаются только 

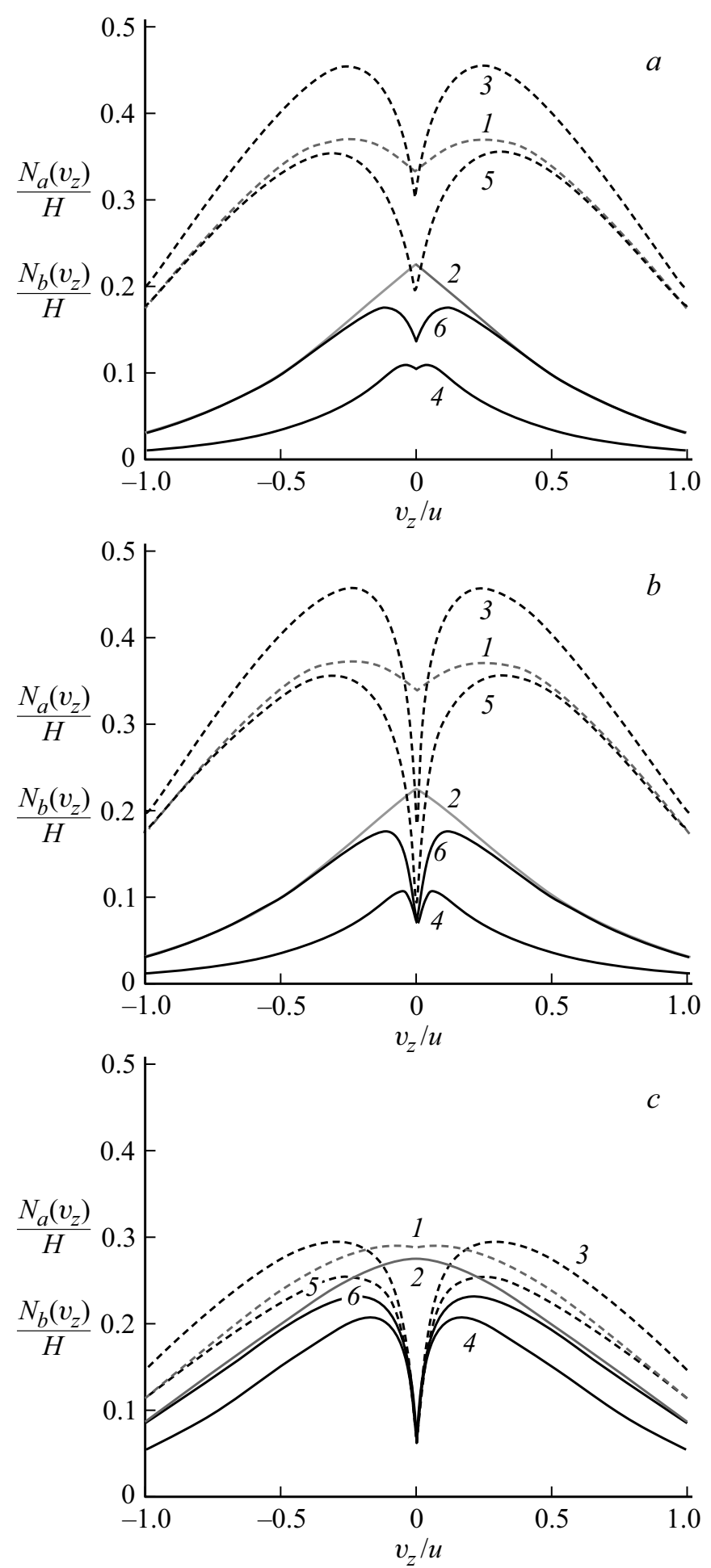

Рис. 3. Эффективное распределение населенностей $N_{a}\left(v_{z}\right)(1,3,5)$ и $N_{b}\left(v_{z}\right)(2,4,6)$ уровней $a$ и $b$ атомов, сосредоточенных в области пробного пучка (рис. 1), по продольной компоненте атомной скорости $v_{z}$, когда величина $H=n_{a} u^{-1}, \gamma_{b} L / u=0.2, A_{b a}=\gamma_{b}(1,2), 0.5 \gamma_{b}(3-6)$, $R / L=10(a), 25(b, c)$. Рис. $3, a$ и $3, b$ соответствуют значениям параметра оптического насыщения $\xi_{a b} / \gamma_{b}=0.5 \quad(3,4)$, $2(1,2,5,6)$, рис. $3, c-\xi_{a b} / \gamma_{b}=5(3,4), 20(1,2,5,6)$. значением отношения $R / L \gg 1$ размеров газовой ячейки (рис. 1). Кривые на рис. 3,c рассчитаны для случая в 10 раз более высокой интенсивности оптической накачки по сравнению с соответствующими кривыми на рис. $3, b$, но при таком же значении $R / L$. Анализ узких симметричных резонансов в области малых значений $\left|v_{z}\right| \ll u$ на рис. 3 позволяет выделить следующие характерные механизмы их возникновения и уширения.

Так, в случае замкнутого перехода $a \rightarrow b$ зависимости $N_{a}\left(v_{z}\right)$ и $N_{b}\left(v_{z}\right)$ имеют соответственно провал и пик в области значений $\left|v_{z}\right| \leq\left(\gamma_{b}+2 \xi_{a b}\right) L$ (кривые 1 и 2 на рис. 3). Действительно, согласно соотношениям предыдущего раздела, именно при данном условии за время свободного пролета $L /\left|v_{z}\right|$ атомов между торцевыми стенками ячейки может произойти заметное насыщение поглощения на циклическом переходе $a \rightarrow b$, которое возрастает с усилением резонансного широкополосного излучения. Поэтому такие резонансы практически исчезают при достаточно интенсивной оптической накачке (кривые 1 и 2 на рис. $3, c$ ). Из сравнения соответствующих зависимостей с одинаковыми номерами на рис. 3, $a$ и $3, b$ видно, что для циклического перехода $a \rightarrow b$ данные резонансы практически не зависят от отношения $R / L \gg 1$ размеров газовой ячейки (рис. 1$)$.

В случае незамкнутого перехода $a \rightarrow b$ в зависимостях $N_{a}\left(v_{z}\right)$ и $N_{b}\left(v_{z}\right)$ возникают гораздо более узкие резонансные провалы с центром в точке $v_{z}=0$ (кривые 3-6 на рис. 3). Данные резонансы обусловлены усилением оптической перекачки населенности из системы уровней $a$ и $b$ (рис. 2) для атомов с ростом указанного времени $L /\left|v_{z}\right|$ их пролета. В случае сравнительно низкой интенсивности оптической накачки $\xi_{a b} \ll \gamma_{b}(11)$ подобные скоростные провалы в населенности $N_{a}\left(v_{z}\right)$ основного состояния $a$, в частности, анализировались в исходной теоретической работе [3]. Очевидно, что подобные провалы возникают также и в населенности $N_{b}\left(v_{z}\right)$ возбужденного уровня $b$ светоиндуцированного нециклического перехода $a \rightarrow b$ (кривые 4 и 6 на рис. 3 ). Для этих резонансов характерно остаточное уширение порядка $(L / R) u$. Действительно, совокупность оптически накачанных атомов в тонкой газовой ячейке является аналогом атомного пучка, расходимость которого определяется отношением $L / R \ll 1$ размеров такой ячейки (рис. 1). Проявление данного уширения видно из сравнения зависимостей 3-6 на рис. 3, $a$ и $3, b$, полученных при различных значениях $R / L$.

Повышение интенсивности накачки приводит к росту как амплитуды, так и ширины резонансных провалов в зависимостях $N_{a}\left(v_{z}\right)$ и $N_{b}\left(v_{z}\right)$ (рис. $3, b$ и $\left.3, c\right)$. Подобное полевое уширение резонансов для нециклического перехода $a \rightarrow b$ (рис. 2) может стать доминирующим при достаточно интенсивной оптической накачке, когда $\xi_{a b} \gg \gamma_{b}$. При этом, согласно соотношениям предыдущего раздела и рис. $3, c$, с ростом значения $\xi_{a b}$ эффективная ширина такого резонансного провала стремится к постоянному значению $\left(\gamma_{b}-A_{b a}\right) L$. Действительно, 


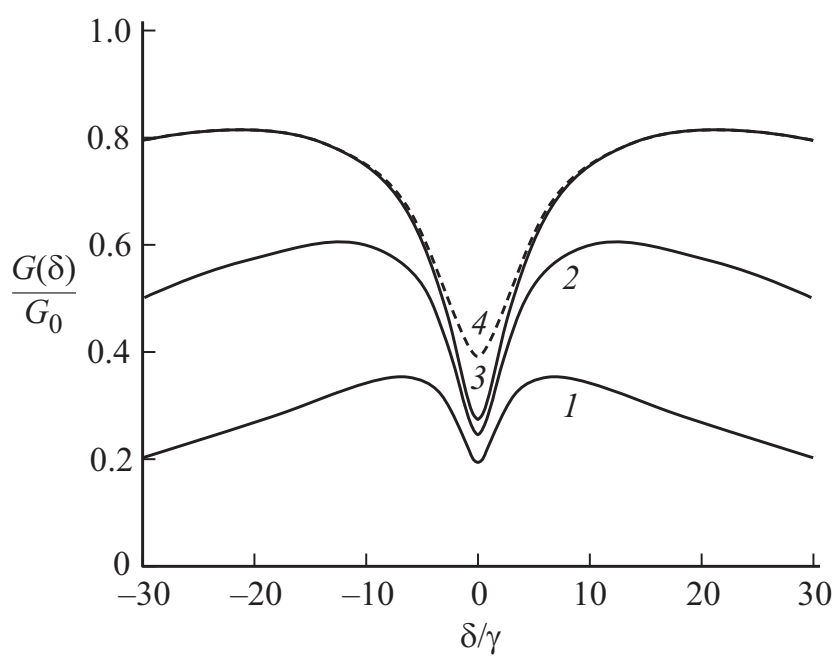

Рис. 4. Зависимость коэффициента поглощения $G(\delta)$ пробного светового пучка от отстройки его частоты $\delta$ для резонансного перехода $b \rightarrow c$, когда $\gamma=0.01 k u, \gamma_{b} L / u=0.2, A_{b a}=0.5 \gamma_{b}$, $R / L=125$ (1-3), $25(4), \xi_{a b} / \gamma_{b}=0.5(1), 2(2), 20(3,4)$.

величина $\left(\gamma_{b}-A_{b a}\right)$ определяет максимальную вероятность (в единицу времени) радиационного распада атома за пределы светоиндуцированного незамкнутого перехода $a \rightarrow b$. Поэтому при сильном оптическом насыщении перехода $a \rightarrow b$ существенное уменьшение населенностей его уровней $a$ и $b$ имеет место для атомов с характерным временем пролета $L /\left|v_{z}\right|>\left(\gamma_{b}-A_{b a}\right)^{(-1)}$. Заметим, что с ростом оптической накачки в области значений $\xi_{a b} \gg \gamma_{b}$ уменьшается также различие между зависимостями $N_{a}\left(v_{z}\right)$ и $N_{b}\left(v_{z}\right)$ (рис. $\left.3, c\right)$.

Рис. 4 представляет зависимости коэффициента поглощения $G(\delta)(12)$ слабой пробной волны от отстройки $\delta=\left(\omega-\omega_{0}\right)$ ее частоты $\omega$ относительно центра $\omega_{0}$ исследуемого квантового перехода $b \rightarrow c$ (рис. 2) с достаточно малой однородной полушириной спектральной линии $\gamma \ll k u$ по сравнению с характерным допплеровским уширением $k u$ этой линии. При рассматриваемых условиях, согласно эффекту Допплера [1], для фиксированного значения $\delta$ основной вклад в величину $G(12)$ будут давать оптически возбужденные на уровень $b$ атомы, у которых проекция скорости $v_{z}$ удовлетворяет условию $\left|\delta-k v_{z}\right| \leq \gamma$. В связи с этим зависимости $G(\delta)$ на рис. 4 подобны соответствующим зависимостям $N_{b}\left(v_{z}\right)$ на рис. 3. В частности, имеет место аналогичный рост амплитуды и ширины внутридопплеровского резонанса с повышением интенсивности накачки, а также уширение таких резонансов при уменьшении отношения $R / L$ размеров газовой ячейки (рис. 1).

\section{Заключение}

В настоящей работе продолжено теоретическое исследование возможностей хорошо апробированного метода внутридопплеровской спектроскопии, соответствующего схеме на рис. 1. При этом в отличие от расчетов предыдущих работ по данной проблеме [3-9] не использовались ограничения (11) на внутреннюю толщину газовой ячейки и интенсивность широкополосной оптической накачки. Таким образом, были проанализированы всевозможные механизмы скоростной селекции атомов на оптически возбужденных уровнях $a$ и $b$, обусловленные пролетной релаксацией данных атомов в рассматриваемых ячейках. На основе этих установленных скоростных особенностей были теоретически исследованы внутридопплеровские резонансы поглощения пробного светового пучка на квантовых переходах типа $b \rightarrow c$ (рис. 2), которые существенно зависят от интенсивности широкополосной оптической накачки и отношения радиуса $R$ газовой ячейки к ее внутренней толщине $L$ (рис. 1). Результаты данной работы могут найти применение в спектроскопии высокого разрешения атомов (молекул) в достаточно разреженной газовой среде, а также для стабилизации лазерной частоты по установленным внутридопплеровским резонансам.

\section{Список литературы}

[1] Demtroder W. Laser Spectroscopy: Basic Concepts and Instrumentation. Springer, 2003.

[2] Riehle F. Frequency Standards-Basics and Applications. Berlin: Wiley-VCH, 2004.

[3] Измайлов А.Ч. // Опт. и спектр. 1993. Т. 75. № 3. С. 664; Izmailov A.Ch. // Opt. Spectrosc. 1993. V. 75. N 3. P. 395.

[4] Tachikawa M., Fukuda K., Hayashi S., Kawamura T. // Jpn. J. Appl. Phys. 1998. V. 37. N 12B. P. L1556.

[5] Otake M., Fukuda K., Tachikawa M. // Appl. Phys. B. 2002. V. 74. N 6. P. 503.

[6] Izmailov A.Ch., Fukuda K., Kinoshita M., Tachikawa M. // Laser Physics. 2004. V. 14. N 1. P. 30.

[7] Imanishi S., Tanaka U., Urabe S. // Jpn. J. Appl. Phys. 2005. V. 44. N 9A. P. 6767.

[8] Fukuda K., Furukawa M., Hayashi S., Tachikawa M. // IEEE Trans. Ultrason. Ferroelectr. Freq. Control. 2000. V. 47. N 2. P. 502.

[9] Fukuda K., Kinoshita M., Tachikawa M. // Appl. Phys. B. 2003. V. 77. N 8. P. 823.

[10] Briaudeau S., Saltiel S., Leite J.R.R., Oria M., Bramati A., Weis A., Bloch D., Ducloy M. // J. de Phys. IV. 2000. V. 10. N Pr-8. P. 145. 